\title{
Introduction: when embodied cognition and sport psychology team-up
}

\author{
Massimiliano L. Cappuccio
}

Published online: 19 February 2015

(C) Springer Science+Business Media Dordrecht 2015

I.

One of the main undertakings of the embodied approach to cognition is to spell out effectively the intuition that our body shapes what our mind can do (Varela, Thompson, and Rosch 1991; Gallagher 2005). This endeavor is motivated - among other things by the deep sense of awe that cognitive scientists experience in front of the sophistication, flexibility, and variability that can be reached by the motor abilities of welltrained humans. In particular, excellence in sporting skills inspires embodied cognition by exhibiting tangible evidence that the details of our bodily constitution are not accidental to our mental powers, but define them in various inherent ways: not only because the informational processes that realize our intelligent functions are specifically regulated, distributed, filtered, or scaffolded by the physical and biological compound that implements them (Shapiro 2011); but also because the modes and the efficacy of our practical intelligence primarily depend on the originary unprincipled engagement with a perceptual environment that can only be discovered by and become meaningful to an embodied agent.

It is the history of the recurrent interactions between body, environment, and social context that defines sophisticated patterns of sensorimotor correspondence, defining at once a worldly horizon of viable opportunities for action and a correlated embodied "self" that is ready to take advantage of these opportunities. Therefore, it is not just metaphorically that we speak of "good feet" and "quick hands" as making a difference in the way a professional football player or a Formula One pilot perceive the world and skillfully cope with it, because the athlete's conceptual understanding and decisionmaking capabilities build first of all on her situated expertise, i.e., on the development of a direct responsiveness to the surroundings, a readiness to anticipate and take advantage of the opportunities of action that the others cannot see. Maurice MerleauPonty (1945) used to characterize this practical, relational know-how as a sort of "tacit knowledge" or "knowledge in the hands", a kind of proficiency that - as opposed to the conceptual knowledge based on explicit instructions, stored heuristics, and amodal representations - relies on the concrete familiarity with practical contexts and personal

M. L. Cappuccio ( $₫)$

United Arab Emirates University, Al Ain, Abu Dhabi, United Arab Emirates

e-mail: m.lorenzo@uaeu.ac.ae 
or interpersonal experience situated in the contingencies of individual or collective performance.

In contrast to the traditional cognitive sciences, which assume that the necessary building blocks of any intelligent function are logical operations of symbolic manipulation (implying the context-neutral transferring of amodal information from an external world to an internal container), the embodied approaches to cognition emphasize the role played by the direct responsiveness to a familiar environment, developed by a situated agent through "unreflective" involvement of a perceptual and motoric kind (Rietveld 2008), without the mediation of stored heuristics, propositional contents, or encoded algorithms: the kind of situated adaptiveness enacted through the body is not only more fundamental than declarative and symbolic intelligence (in fact, the kind of sport skills mastered by the athletes don't seem to presuppose the actual capability or even the theoretical possibility to analytically describe how they work); it is also pervasive in and constitutive of "higher" and more intellectually detached cognitive faculties (e.g., propositional judgment, conceptual categorization), to the point that the latter struggle to develop if the former is deficient.

The theory of embodied cognition resonates at least in part with some of the psychological doctrines situated at the root of the sport sciences, e.g., both the cognitive-computational approach by Fitts and Posner (1967) (which stresses how bodily engagement with the environment provides specific tools for perceiving, remembering, thinking, and acting that can hardly be replaced by "intellectual" skills on their own) and the cognitive-ecological approach by Gibson (1979) (maintaining that the physical constitution of our body carries out a significant portion of the information-processing traditionally attributed to mentation). These psychological doctrines are deeply implanted in the everyday research practice of the sport sciences; that is why it is not surprising that the most influential theories of embodied cognition begin to be seriously addressed (though not universal accepted at all) by many researchers in sport psychology.

This interest is testified by the work of leading performance scientists like Thomas Carr and Sian Beilock: thanks to them and their colleagues, it is today documented how well-trained athletic skills correlate in expert performers with fine perceptual abilities, fast recognition, robust visualization and mental imagery, rich linguistic mapping, accurate categorization, effective tactical understanding, reliable predictions, and efficacious decision-making. These empirical studies were reviewed by Moreau (this volume) and, before that, in Sian Beilock's seminal paper (Beilock et al. 2008), where it is argued that "(a) the ability to perceive and predict the actions of others; (b) the ability to comprehend action-related language; and (c) the judgments individuals make about objects and events in their environment" arise from and are modulated by "the variations in individuals' motor skill repertoires". Both Beilock and Moreau also relate this idea to various influential neuroscientific studies on the motor and pre-motor cortex, building on the authoritative line of research developed by Giacomo Rizzolatti and his school during the last twenty years (Rizzolatti et al. 2001). The convergence of behavioral data, psychological models, neuroscientific findings, anecdotal reports, and phenomenological accounts not only confirm that mental abilities traditionally characterized as "intellectual" or "cognitive" (e.g., mental toughness, focus, visualization) are highly relevant to the performances of the expert athletes; conversely, it also suggests - in accord with the principles of embodied cognitive 
science - that athletic skills exhibit their own characteristic intelligence: a non-conceptual, pre-linguistic capability that doesn't rely on acontextual rules and apriori models, being rather normed by the motoric and perceptual contingencies of executive control and real-world interactions.

As highlighted by Beilock (2008), there are important common motifs and aspirations that envisage the convergence between the empirical studies of sport psychology and the embodied approach in the cognitive and brain sciences. That their mutual correspondence is strong is becoming clear to more and more successful sport psychologists, who recognize that the results and the models of embodied cognitive science can suggest more effective training methods; in a reciprocal way, attentive cognitive scientists can't overlook sport and exercise psychology, as this field is one of the richest terrains for empirical exploration, experimental discovery, and epistemological validation of models and theories (cf. Sutton 2007). The cooperation between these two scientific fields promises immediate and tangible translational benefits: it doesn't only provide concrete methods to try the theoretical models, testing how effectively they can help improve the athletes' agonistic results; it also raises the epistemological value of the sport science, as it documents how the empirical study of athletic performance helps recognize the specific patterns of various embodied faculties and isolate the hidden variables that underlie their functioning. Scientific cooperation is facilitated by the fact that most of the explanatory models of the athletes' motoric and perceptual competences can be tested and falsified, either in real-world competitive settings or in controlled environments during opportunely designed laboratory studies: in either case, sport performances provide case studies that are paradigmatic to the methodology of embodied cognition in that, by their own competitive nature, sport tasks are designed for testing and measurement in neutrally reproducible settings. These are the invaluable theoretical insights that sport can offer to the sciences of mind, concerning how skills are actually enacted and controlled, through the body, defining dynamical boundaries between mind and world.

II.

But what is exactly an athletic skill, and how does it deploy the kind of situated intelligence studied by embodied cognition? This special issue of Phenomenology and the Cognitive Science tries to answer this question from a particular perspective, i.e., investigating what happens when sporting skills stop working and unreflective knowhow is temporarily impaired: strikingly, it is when our routine coping skills break down that the very normative structure of intelligent action becomes most perspicuous. In this spirit, this collection of papers is meant to recapitulate, compare, and possibly expand, through the help of the phenomenologically oriented cognitive sciences, the explanatory models of the debilitative phenomenon called "choking under pressure" or the "choking effect" (Beilock 2011). This phenomenon is related to a multifaceted complex of cognitive, motivational, and emotional dynamics that are largely studied-but diversely defined - by sport psychologists (Mesagno 2013; Mesagno and Hill 2013). While the scientific definition of choking may vary significantly, and there is no universal agreement about its nature and causes, the term refers to a common paradox many practitioners have to become familiar with in their everyday life: expert athletes often produce below-standard performances (suddenly failing even routine tasks) just during competitions that over-emphasize the expectation to produce excellent results. 
In the sport sciences, at least three empirical models compete to explain why this catastrophic phenomenon occurs so often in tournaments or other pressure-filled situations: distraction (Wine 1971), overload (often conflated with distraction), and self-monitoring (Beilock and Carr 2001) theories. They impute the negative effects of choking to the intrusion of external competing attentional stimuli, the overflowing of internally available computational resources, or the disruption of fluid habitual motor routines, respectively. Note that, while the contingent causes indicated by these models can sometimes partly overlap, it is useful to distinguish them: in fact, distraction is not necessarily caused by overload, whose origin and phenomenology are in turn different from self-monitoring. Further explanatory narratives are also possible: for example, it is possible to interpret over-arousal as a distinct source of choking, one that specifically originates within emotional and motivational systems, and thereby perturbs the physiological performance environments of cognitive systems in addition to stimulating alterations in choice of cognitive strategies (Carr, this volume); also, it is possible to understand distraction sensitivity, the difficulty level of the task at hand, and its strategic requirements as three interdependent parameters in an integrated attentional system that is common to both habitual and cognitive action (Christensen et al., this volume).

Carr, Beilock, and their fellow colleagues have conducted numerous studies on what they call "automated" sensorimotor skills, understood as habitual action routines that, even if produced without explicit control or reflection, are not necessarily stereotyped, inflexible, and unintelligent nonetheless (e.g., Beilock et al. 2002a, 2004, 2008; Beilock and Gonso 2008). While the results of these experiments have been differently interpreted, they have certainly been influential in corroborating the arguments in favor of the third model of choking (self-monitoring) and systematically exposing the limits of the other two (without entirely excluding them). That is one reason why selfmonitoring theories are so central in the present collection, offering either a reason of inspiration or a target of critical reflection to most of the contributions included here.

What are the most important arguments in favor of the self-focus theory of choking? First of all, the aforementioned authors maintain that distraction theories don't account for the fact that choking typically occurs in conditions of total concentration, while it normally doesn't affect experts in conditions of split attention (as in concurrent dual tasks, such as performing a golf putt while being asked to simultaneously recognize a given tone); also, they argue that cognitive overload theories don't account for the fact that choking doesn't normally affect experts who perform in fast action conditions (under speed instructions of the kind "putt as quick as possible"), while self-paced action conditions (under attention instructions such as "putt only when you think you are ready") typically increase their chances of choking. In turn, self-monitoring theories account for the low level of expert performances in the dual task conditions in which the secondary task demands attentive analysis of the primary motor tasks (e.g., "tell when the swing movement is finished"), or under attention instructions that stress the component processes of the performance (e.g., "keep the knees bent"); additionally, self-monitoring theories usually do better in explaining why these conditions have a negative effect only on expert athletes performing well-practiced sensorimotor skills, while the novices typically exhibit a drop in performance under the conditions that don't damage the experts (i.e.,: dual-tasks without self-monitoring and speed instructions); last but not least, how could the other theories explain that instrumentally 
produced distraction is customarily used as an effective expedient to prevent choking, building on the evident fact that external foci can force the athletes to stop overscrutinize the details of their own movements? (Jackson et al. 2006; Wulf and Su 2007).

Taken together, these bodies of evidence can be used to argue that the disruption of the fluid course of familiar automatized routines is due to self-monitoring (or, more precisely, "execution focus", as elucidated by Carr, this volume), which is the reason why choking is often defined also as "paralysis by awareness/analysis" (Jackson and Beilock 2008). The main assumption underlying their theory is that performance anxiety typically solicits self-monitoring in the athletes during competition; self-monitoring, in turn, is likely to disrupt the continuous flow of the action by breaking its holistic and highly organic structure into a mechanic array of inflexibly juxtaposed segments of movements. However, this picture does not solve all the issues yet: to begin with, not everybody sees a necessary causal link connecting anxiety to selfmonitoring (e.g., Montero, this volume); also, conflicting opinions can arise about the cognitive requirements necessary to obtain the coherence and fluidity of complex movements, if simply attending to one's own sensorimotor activity is sufficient to decompose it in a way that undermines its own effectiveness and adaptivity.

Choking poses to both science and philosophy a fascinating puzzle exactly because any attempt to model it raises this kind of foundational questions on the nature of action, asking us to examine our theoretical assumptions on the normativity of skills and embodied intelligence. Any theory of choking is contingent upon some specific interpretation of the nature of the sensorimotor skills that underlies it. This is of course true also of the self-monitoring theory, whose most important theoretical assumption is that what makes fluid, quickly adaptive, and richly flexible the live action of an expert is the fine-grained arrangement of its kinematic elements into a systemic and temporally unitary composition, so complex - and, at the same time, so harmoniously organized - that the expert cannot be explicitly aware of it while executing it. Similar considerations must be at the basis of another important view in sport psychology, the "constrained action hypothesis" (Wulf et al. 1999; Wulf, Shea, and Park 2001), which asserts that the attentive monitoring of one's own action, with the subsequent decomposition of the harmonious routines into their component processes, may result into the mechanical production of inelegant and scarcely flexible movements: this is why attempts to consciously analyze one's own movements (internal focus) disrupt the functioning of the motor system by interfering with automatic control processes; while, interestingly, attention to the immediate consequences of those movements (external focus) doesn't produce this negative effect (Wulf, Shea, and Park 2001).

Embodied cognition theory deeply resonates with this idea and provides its perfect background: as importantly observed by Powers (1978), competent goal-directed action involves monitoring one's perceptions of how the world is changing as one acts, not the actions themselves; and, interestingly, similar ideas were subsequently elaborated in various flavors by the sensorimotor theories of consciousness and enactive cognition theory (Varela, Thompson, and Rosch 1991; O’Regan and Noë 2001; Noë 2004; Thompson 2007). Powers' perceptual control theory maintains that the skill of a performer consists in making - with her actions - the world look a certain way, as it is how the world is changing (toward or away from the desired goal state) that constitutes the information that guides action in real-time and allows adjustment to the immediate changes. The full acquisition of automaticity in these sensorimotor 
feedback loops, which is possible when the on-line updating of the body-world configurations brings about a dynamic coupling between the agent and its cognitive environment, is exactly what allows for reliable predictability and flexible adaptivity in our skillful sensorimotor performances.

It is also possible to characterize in a stronger speculative sense the theory of choking as disruption of automaticity: according to such an interpretation of the theory, if choking only affects expert performers who have already automatized their actions this is because the athletes not only can but, to some extent, must rely on automatized routines to achieve optimal performances, under the assumption that automatization is the distinctive mark of a well trained and confident skill. The fact that the novices have not assimilated these routines yet, on the other hand, usually means exactly that they need to pay constant attention to the component processes of their actions in order to produce average performance; they cannot be significantly affected by paralysis because they always already are in a structural condition of awareness/analysis when they perform. In this particular sense, to the supporters of this stronger version of the selfmonitoring theory, these two dichotomous couples (expert/novices and automatic/selfmonitoring action) seem to overlap very nicely, as the boundary that define their respective meanings is by and large the same. Some experimental data proving a substantial differentiation in the nature of the cognitive processes underlying skillful performances could be cited in favor of the hypothesis that skill level is generally proportional to the capability to produce complex motor output without conscious control: compared to the novices, the experts rely more on their procedural memory than their declarative memory to remember what happens during performances (Beilock et al. 2002b); that is why both their superior skills and their sensitivity to choking depend on how confidently they rely on rich and sophisticated motor routines that don't need conscious control (Beilock, Wierenga, Carr 2002; Beilock and Carr 2004).

For the readers of Phenomenology and the Cognitive Sciences it is particularly interesting that some phenomenologically inspired philosophies of action seem to go hand in hand with this strong interpretation of choking as self-monitoring: probably, the most influential is Hubert Dreyfus' phenomenological doctrine of skillful action, which is paradigmatic in asserting that an agent masters a embodied skill only when her online responses to the changes in the environment occur as "absorbed coping", which means - among other things - that they are fully automatized (Dreyfus 2002, p. 370). It is worth reminding ourselves again that, in this context, "automatic" doesn't mean reducible to a mechanical procedure governed by a fixed model or a predefined script but, on the contrary, fluidly embedded in the agent's holistic spontaneous dispositions towards immanent sensorimotor circumstances, without awareness of reflection being involved. That is why what defines expertise in the context of Dreyfus' theory (and other approaches inspired by Merleau-Ponty) is exactly that the expert's action is not guided by step-by-step instructions, explicit rules, or decisional procedures, but by a fine-grained sensitivity to complex configurations of contextual contingencies: in its most paradigmatic form, expert action occurs with neither decision nor control.

This phenomenology of absorbed coping builds on a very influential empirical report on the nature of skill acquisition that Dreyfus accomplished with his brother for the US Air Force. In Dreyfus and Dreyfus (1980) they conclude that maturing mastery in a certain skill (after passing through the intermediate stages of novice, competent, proficient, and expert), corresponds precisely to replacing explicit rules with 
tacit knowledge: attention to the component processes of one's action progressively dissolves as the execution becomes more absorbed, while on the other hand attending to the details of one's action, with explicit awareness of the rules that govern its course, is the distinctive mark of apprenticeship, when the novice is still in the process of incorporating into an implicit know-how the particular heuristics he uses for making particular decisions and solving particular problems: according to this view (challenged by Hutto and Sánchez-García, this volume), optimal training sessions are accompanied by constant self-monitoring and attention to component processes, while optimal performances spontaneously spring from thoughtless and almost unaware automaticity. According to this analysis, Dreyfus states that skillful performances necessarily arise from a state of deep absorption into the perceptual contingencies of one action. Some critical commentators (e.g., Zahavi 2012) have remarked that the kind of absorption described by Dreyfus does not simply exclude consciousness; it must be "mindless" too, in that it presupposes the vanishing of subject/world distinction. Dreyfus's theoretical foundation of absorbed coping builds on an adamantly anti-representationist narrative: representation of one's own action, i.e., awareness of its rules and normative preconditions and attention to its component processes, doesn't and can't play any positive role in true expertise, and actually emerges only when the agent's absorption fades away or is not yet ready. In this perspective, representation comes either before skill acquisition or after skillful performance, i.e., it is either a temporary scaffold necessary to automatize a routine (as during training), or a conceptual expedient to rationalize its defining principles a posteriori. Before and below an explicit, declarative, "intellectual" or "cognitive" form of intelligence, based on the mediation of models, plans, rules of thumb, problem-solving procedures, detached decisional processes, and extrinsic judgments formulated to explicitly represent an objective situation, there is another, more fundamental, inherently practical, embodied form of intelligence, which is purely based on direct responsiveness to familiar opportunities for action (Dreyfus 1996). This intelligence is an expert system's sensitivity to the relevant environmental fluctuations, expressed by the living body's capability to fluidly counterbalance the contingencies. Such responsiveness is what allows us to spontaneously perceive sensorimotor contingencies as meaningful affordances. According to Dreyfus (and other theorists who, like him, build on system dynamics models such as Freeman 1999), embodied intelligence is inherently nonrepresentational precisely because it builds on such expert interactive dispositions matured in a history of sensorimotor coupling between body and environment, incorporated in the system as an implicit adaptive capability aiming at approximating a dynamical equilibrium with the contextual contingencies. However, the issue on whether this kind of adaptive coupling necessarily excluded representation is currently hotly debated among philosophers of mind, and the articles by Gottlieb and by Hutto and Sánchez-García included in this collection can well testify how different the approaches to this issue can be.

The question about whether the architecture of the mind is representational or not itself is closely tied to the issue concerning whether the mind of an expert is active or passive in relation to its ecological situation. Even if many of the contributions presented in this collection endorse (at least to some degree) some version of the thesis that the nature of intelligent skills is fundamentally embodied, and even if the antirepresentationist option is well represented by the articles by Hutto and Sánchez-García and by Ilundáin-Agurruza, their authors (as well as many other working in this field) have reservations towards Dreyfus's conclusion that a "mindless" (i.e., unaware and 
essentially passive) absorption is a necessary requirement to expertise. Some of these reservations are motivated by the many exceptions that empirically contradict the model of absorbed coping: the characterization of the expert as acting almost unconsciously, without decision or explicit control, fails in varying degrees when the expert is either dealing with an unusual task situation or unusual task demands, or when he is explicitly trying to repair a fault in execution that has somehow developed (as when Tiger Woods is "rebuilding" his swing); but also-according to Christensen et all. (this volume), and Sutton (2007) when the task itself requires presence of mind and explicit awareness of the athlete's own body.

There is also another reason for skepticism, related to the specific features of Dreyfus's anti-representationalism: in the literature it is not difficult to find several attempts to fully reject it, revise it, or at least complete it (for example, Sutton et al. 2011. Or Cappuccio and Wheeler 2012, which offers an attempt to introduce minimal elements of representationalism within the Dreyfusian conventional discourse on the cognitive background of skillful coping). The main reason of disagreement is that, while most theorists of embodied and enactive cognition find it plausible that a practical know-how entirely relying on adaptive dispositions doesn't require representation, Dreyfus uses this principle to justify the much more demanding conclusion that representation and expert skill are mutually exclusive. This is because Dreyfus conflates attention and analysis under the category of representation (this term, in his perspective, encompasses both awareness and reflection) and, rejecting representation as a possible element of embodied skill (a term that indicates, in Dreyfus's habitualist perspective, a familiar routine), concludes that not only self-monitoring, but any kind of explicit attention to the particular circumstances of the performance, must be avoided by the expert performer.

Of course it is possible to try to resist this conflation: in fact, if Dreyfus were right, then how could we make sense of the instances of aware reflection deliberately used by skillful performers (and especially and most effectively by the experts) to plan, guide, or correct their actions? Should we perhaps conclude that skilled athletes are not really performing "as experts" at the time they pay attention to what they are doing (a conclusion that Dreyfus is deemed to favor)? Or should we adopt different notions of "skill" or "representation"?

Certainly, this set of closely related questions, which is central to the collective endeavor that motivates this special issue, can be answered by a multiplicity of diverse theoretical angles. In general, while some authors are more skeptical than others about Dreyfus's conclusion, their skepticism can be motivated by very diverse reasons: for example, some of them - like Dreyfus - assume that representation inhibits performances, but - unlike him - reject the idea that self-focus is a necessary component of representation; while other simply disagree with the statement - shared by both Dreyfus's philosophical theory and the self-monitoring theories in sport psychology that representation is necessarily detrimental to performance, tout court. The research papers that form this collection reflect this rich diversity of points of view, exhibiting how unexpectedly complex the patterns of agreement and disagreement that intersect the fields of both cognitive philosophy and sport psychology can be.

The collection is opened by Carr's review, which constitutes an authoritative summary of over twenty years of psychological research on choking carried out by him and his school. His account primarily imputes choking to "execution focus", but it 
is so comprehensive to embrace in a unique multidimensional explanatory model various reasons why skillful action is not always damaged by aware attention. In fact, this is a model that reflects the phenomenological, cognitive, and emotional complexity of this phenomenon, stressing the fundamental role of automaticity in skillful action execution, but also the fact that skill level makes a difference also when the athlete is not "in the zone", i.e., when reflection is necessary to modulate performance in a creative way, or repair troubled and problematic executions.

Execution focus and the other self-monitoring theories of choking don't satisfy the critical analyses presented by other researchers: in particular, Christensen, Sutton, and McIlwain, see their underlying logic troubled by non-sequiturs and ambiguities. Their extensive essay is the outcome of a systematic investigation that considers both qualitative studies (which highlight how attentive presence is key to performance in many sports) and quantitative models based on experimental evidence (which, according to the authors, highlight the inconsistency and the lack of ecological validity of the self-monitoring model of choking). This investigation is meant to highlight how both execution focus and distraction theories are insufficient: a complete new theory of choking is proposed by the authors to explain why control plays a central role in advanced skill, with cognitive and automatic processes being closely integrated.

If the first two contributions are dedicated to the psychological models of choking, Papineau's investigation illustrates, inside the domain of philosophy of mind, a possible foundation for this empirical research: first of all, it reminds us the differentiation between habitualist and intellectualist approaches to skillful performance, and then examines how each of them captures only a particular set of features of performance disruption due to emotional distress. This analysis is enlightened by the conceptual distinction between the control operated at the level of physical reflexes, as opposed to the higher-level intentions that constantly shape the athlete's actions and decisions at the personal level of his mental activity. The author argues that the performance disruption usually called "choking" requires a more precise linguistic description to reflect this duality, as competition anxiety is just one of the ways in which mental fragility can hinder performances: that is why Papineau relates the specific mental dynamics underlying choking to a general loss of focus due to anxiety, distinguishing this problem from other phenomena such as the so-called "yips", which properly captures the paralysis produced by self-monitoring.

The paper by Hutto and Sánchez-García nicely testifies how the collaboration between a philosopher and a sport psychologist can produce a fruitful reinterpretation of the existing theoretical models, advancing a high-profile understanding of the concrete sporting practices. In fact, by building on the 'radically enactive approach to cognition', which strongly de-emphasizes the role of representations to characterize the ecological dimension of skill acquisition, the authors stress that not only performance can be hindered by explicit focus on the action control, but that also skill acquisition suffers if it is based on a linear pedagogy based on the passive imitation of given models and predefined action schemata. After criticizing the limits of a skill learning method based on the mechanical internalization of rules and explicit instructions, the authors propose an alternative, dynamicist approach to skill and training, one that, from the beginning of the learning process, aims at developing adaptive 
capabilities and behavioral norms on the basis of the direct unprincipled engagement of the novice with real live action and creative practical problem-solving.

The role of aware visual consciousness in sport performances is the target of the paper by Koedijker and Mann, who have conducted an outstanding line of original empirical research during the last years. Building on a neat methodological distinction between the perceptual and the motoric components of sporting skills execution, the authors argue that the former is both benefitted by expertise and impaired by choking in ways that are analogous to the latter, but also specific and particular to how the gazing patterns are produced and controlled by the agents. The experimental findings reviewed in this article show that visual skills, exactly like executive performances, are facilitated by expertise, can be successfully enhanced by means of specific training, and can possibly fail, when pressure assails the athlete.

Also the article by Moreau offers a rich review of the experimental literature, even though his perspective is one that highlights the overlaps and the original correlation between visual and motoring competences. Its purpose is to document how various findings in sport psychology supports the general view of embodied cognition, testifying how motor skills and expertise are not simply modulated by explicit thought and imagery, but also - in turn - actively modulate various cognitive processes on their own, improving the capabilities of perceptual recognition and categorization that underlie our spatial intelligence. The author addresses the neurophysiological architecture of these skills, endorsing the view that the competences enacted by the motor areas of the brain are re-used for other cognitive tasks, hence providing an embodied foundation to more intellectual forms of cognition.

One of the main theoretical issues in today's cognitive philosophy and in this collection is the discussion of whether representation plays a constitutive role for our mental life. The paper by Gottlieb centrally addresses this issue, examining and comparing what the models of skillful action can do with or without the notion of representation. In this context, Dreyfus' dynamicist model is explicitly contrasted with Carr's, Beilock's, and Masters' model, which — in spite of the evident analogies with Dreyfus's ideal of absorbed coping - still relies on the notion of representation. The author takes a stand in relation to this opposition, and develops a line of argument against the Dreyfusian attempt to conflate representation with attention and reflection, and asserting that some categories of action do actually rely on a representation of rules and models.

The article by Montero challenges the model of choking as paralysis produced by self-monitoring, building on arguments inspired by distraction theories and a critical discussion of the methodological background of Carr's and Beilock's theories. She proposes a combination of detailed conceptual analyses and anecdotal evidence aiming at showing how the execution focus model still needs ecologically valid empirical data to be eventually confirmed, and that the role of emotions in the generation of choking has not been fully clarified yet. In particular, she stresses that the causal link between anxiety and self-monitoring still has to be proven, and that mental models and explicit recollection of step-by-step instructions is beneficial to performance and can even prevent paralysis.

The collection is closed by the article by Ilundáin-Agurruza, which relates the psychological studies on choking to both the phenomenological tradition and the oriental doctrines of martial preparation. The author focuses on the experiential value 
of choking as a revelatory phenomenon that discloses the original meaning of anxiety as tragic anticipation of death. Building on an insightful examination of the traditional Japanese values of "dō", as embedded in various arts of self-cultivation, and recognizing their theoretical valence in the light of the existentialist and pragmatist motifs developed by phenomenologal research, the author invites us to interpret anxiety not just as an obstacle to optimal performances, but as an opportunity for maturation, motivated by the search for one's own authentic balance with herself and the environment.

One question (simple to formulate, but difficult to answer) seems to emerge throughout the reading of these contributions: apparently, some forms of self-monitoring, attentive control, and mental presence consistently produce choking, while other forms of these phenomena are not detrimental or are even necessary to skillful performance; if that is the case, then what is the real element of differentiation between the former and the latter?

It is possible that, in order to propose a more comprehensive theory of choking that could answer it, we would need additional empirical data, especially to detail how the general mechanisms responsible for cognitive deterioration are causally related to individual psychological differences and personal predispositions, which might for example involve emotional complexes or motivational narratives. In turn, this fundamental step in the psychological research requires a closer discussion of the epistemological, ontological, and phenomenological background of the currently available psychological models. Definitively we will need more interaction between cognitive philosophers and sport psychologists in the future, to complete or re-interpret the available concepts of skill acquisition and skill disruption.

Acknowledgments This special issue of Phenomenology and the Cognitive Sciences represents the outcome of an interdisciplinary research project sponsored by a 2013-14 grant by the National Research Foundation of the United Arab Emirates and UAE University, with the collaboration of Abu Dhabi Sports Council. In my capacity of principal investigator of this project, I am thankful to Sami Boudelaa for his role of senior guidance in our research partnership and for his wise recommendations, and to my respected colleagues, Mohamed Al-Baili, Nasser Al-Ameri, Steve Bird, Ali Al-Ghafli, Ateeq Jaka, William McDonald, Fdwa Al-Mughairbi, Jamal Al-Nuaimi, Saif Al-Qaydi, for encouraging and sustaining this seminal line of research in the Arab Gulf. Also, as guest editor of this special issue, I am personally grateful to the Philosophy Department of the University of Memphis for hosting the initial stage of this research, conducted in 2012 in Tennessee thanks to a Fulbright visiting fellowship. Two academic events importantly prepared the collective process of ideation and realization of this special issue: a symposium entitled "Embodied cognition and sport psychology: From fast motor actions to the choking effect", hosted in July 2013 in Granada during the annual meeting of the European Society for Philosophy and Psychology, and the four-day long "First International Conference in Sport Psychology and Embodied Cognition", hosted in February 2014 in Al Ain by UAE University, with the patronage of Abu Dhabi Sports Council, the College of Humanities and Social Sciences, and the College of Education. I want to express my gratitude to all the researchers and practitioners who participated in these events, contributing to their realization with both their intellectual work and their invaluable experience on the field. Among the others, I must mention at least: Benjamin Aguda, Tom Buchanan, Roland Carlstedt, Alberto Cei, Matthew Cullen, Caren Diehl, Jesús Ilundáin-Agurruza, Martin Kramar, Tom Loney, David Mann, Tadhg MacIntyre, Mauro Maldonato, Fraser McLaughlan, Thomas Patrick, Gareth Picknell, Zuzanna Rucinska, Mark Scott. I would like to thank the authors of the papers comprised in this collection for believing in this project and dedicating their work to its success, and all the colleagues who generously offered their voluntary help and their time to the realization of this special issue: Chiara Brozzo, Ellen Fridland, Tasneem Alsayyed, Robert Kubala, Sara Parmigiani, Angelica Kaufmann, Silvia Grasso. I wish this research at the intersection of embodied 
cognition and sport psychology, and my future work in this field, could always continue to benefit from the precious directions of the distinguished scholars I had the honor to learn from during the last few years: Tom Carr, Shaun Gallagher, Dan Hutto, David Papineau, John Sutton, and Dan Zahavi.

Massimiliano Cappuccio

Al Ain, Emirate of Abu Dhabi

November 2014

\section{References}

Beilock, S.L. (2011). Choke. The secret of performing under pressure, Constable \& Robinson, Edinburgh.

Beilock, S. L., \& Carr, T. H. (2001). On the fragility of skilled performance: what governs choking under pressure? Journal of Experimental Psychology. General, 130(4), 701-725.

Beilock, S. L., \& Carr, T. H. (2004). From novice to expert performance: Memory, attention and the control of complex sensori- motor skills. In A. M. Williams, N. J. Hodges, M. A. Scott, \& M. L. J. Court (Eds.), Skill acquisition in sport: Research, theory and practice (pp. 309-328). London: Routledge.

Beilock, S.L., Gonso, S. (2008). Putting in the mind versus putting on the green: Expertise, performance time, and the linking of imagery and action. The Quarterly Journal Of Experimental Psychology 1-13.

Beilock, S. L., Carr, T. H., MacMahon, C., \& Starkes, J. L. (2002a). When paying attention becomes counterproductive: impact of divided versus skill-focused attention on novice and experienced performance of sensorimotor skills. The Quarterly Journal of Experimental Psychology, 8(1), 6-16.

Beilock, S. L., Wierenga, S. A., \& Carr, T. H. (2002b). Expertise, attention, and memory in sensorimotor skill execution: Impact of novel task constraints on dual-task performance and episodic memory. The Quarterly Journal of Experimental Psychology, 55A(4), 1211-1240.

Beilock, S. L., Bertenthal, B. I., McCoy, A. M., \& Carr, T. H. (2004). Haste does not always make waste: expertise, direction of attention, and speed versus accuracy in performing sensorimotor skills. Psychonomic Bulletin \& Review, 11(2), 373-379.

Beilock, S. L., Bertenthal, B. I., Hoeger, M., \& Carr, T. H. (2008). When does haste make waste? speedaccuracy tradeoff, skill level, and the tools of the trade. Journal of Experimental Psychology, 14(4), 340352.

Cappuccio, M., \& Wheeler, M. (2012). Ground-level intelligence: Inter-context frame problem and dynamics of the background". In Z. Radman (Ed.), Knowing without thinking. Mind, action cognition and the phenomenon of the background. USA: Palgrave Macmillan.

Dreyfus, H., (1996). The current relevance of Merleau-Ponty's theory of embodiment. The Electronic Journal of Analytic Philosophy 4

Dreyfus, H. (2002). Intelligence without representation-Merleau-Ponty's critique of mental representation phenomenology and the cognitive sciences. Netherlands: Kluwer Academic Publishers.

Dreyfus, S., \& Dreyfus, H. (1980). A five-stage model of the mental activities involved in directed skill acquisition. Washington, DC: Storming Media. Retrieved June 13, 2010.

Fitts, P. M., \& Posner, M. I. (1967). Human performance. Belmont: Brooks/Cole.

Freeman, W. J. (1991). The physiology of perception. Scientific American, 264, 78-85.

Gibson, J. J. (1979). The ecological approach to visual perception. Boston: Houghton Mifflin.

Jackson, R. C., \& Beilock, S. (2008). Attention and performance. In D. Farrow, J. Baker, \& C. MacMahon (Eds.), Developing elite sports performers: Lessons from theory and practice. New York: Routledge.

Jackson, R. C., Ashford, K. J., \& Norsworthy, G. (2006). Attention focus, dispositional reinvestment, and skillful motor performance under pressure. Journal of Sport and Exercise Psychology, 28, 49-68.

Merleau-Ponty, M. (1945). Phénoménologie de la perception. Paris: Gallimard.

Mesagno, C. (2013 ed.). International Journal of Sport Psychology_-"Performance under pressure", 44(4).

Mesagno, C., Hill, D.M. (2013). Definition of choking in sport: re-conceptualization and debate, in C Mesagno, International Journal of Sport Psychology, 44 (4)_-"Performance under pressure", 267-277.

Noë, A. (2004). Action in Perception. MIT Press.

O’Regan, J. K., \& Noë, A. (2001). A sensorimotor account of vision and visual consciousness. Behavioral and Brain Sciences, 24(5), 883-917.

Power, W. T. (1978). Quantitative analysis of purposive systems: some spadework at the foundations of scientific psychology. Psychological Review, 85(5), 417-435.

Rietveld, E. (2008). Situated normativity: the normative aspect of embodied cognition in unreflective action. Mind, 117(468), 973-1001. 
Rizzolatti, G., Fogassi, L., \& Gallese, V. (2001). Neurophysiological mechanisms underlying understanding and imitation of action. Nature Reviews Neuroscience, 2, 661-670.

Sutton, J. (2007). Batting, habit and memory: the embodied mind and the nature of skill. Sport in Society, 10(5), 763-786.

Thompson, E. (2007). Mind in life: biology, phenomenology, and the sciences of mind. Cambridge: Harvard University Press.

Varela, F., Thompson, E., \& Rosch, E. (1991). The embodied mind: Cognitive science and human experience. Cambridge: MIT Press.

Wine, J. (1971). Test anxiety and direction of attention. Psychological Bulletin, 76, 92-104.

Wulf, G., \& Su, J. (2007). An external focus of attention enhances golf shot accuracy in beginners and experts. Research Quarterly for Exercise and Sport, 78, 384-389.

Wulf, G., Lauterbach, B., \& Toole, T. (1999). Learning advantages of an external focus of attention in golf. Research Quarterly for Exercise and Sport, 70, 120-126.

Wulf, G., Shea, C. H., \& Park, J.-H. (2001). Attention in motor learning: preferences for and advantages of an external focus. Research Quarterly for Exercise and Sport, 72, 335-344.

Zahavi, D. (2012). Mindedness, mindlessness and first-person authority. In J. Schear (Ed.), Mind, reason and being-in-the-world: The Mcdowell-Dreyfus debate. London: Routledge. 